\title{
Phylogenetic analysis of erythritol catabolic loci within the Rhizobiales and Proteobacteria
}

\author{
Barney A Geddes, Georg Hausner and Ivan J Oresnik
}

\begin{abstract}
Background: The ability to use erythritol as a sole carbon source is not universal among the Rhizobiaceae. Based on the relatedness to the catabolic genes in Brucella it has been suggested that the eryABCD operon may have been horizontally transferred into Rhizobium. During work characterizing a locus necessary for the transport and catabolism of erythritol, adonitol and L-arabitol in Sinorhizobium meliloti, we became interested in the differences between the erythritol loci of S. meliloti and R. leguminosarum. Utilizing the Ortholog Neighborhood Viewer from the DOE Joint Genome Institute database it appeared that loci for erythritol and polyol utilization had distinct arrangements that suggested these loci may have undergone genetic rearrangements.

Results: A data set was established of genetic loci containing erythritol/polyol orthologs for 19 different proteobacterial species. These loci were analyzed for genetic content and arrangement of genes associated with erythritol, adonitol and L-arabitol catabolism. Phylogenetic trees were constructed for core erythritol catabolic genes and contrasted with the species phylogeny. Additionally, phylogenetic trees were constructed for genes that showed differences in arrangement among the putative erythritol loci in these species.

Conclusions: Three distinct erythritol/polyol loci arrangements have been identified that reflect metabolic need or specialization. Comparison of the phylogenetic trees of core erythritol catabolic genes with species phylogeny provides evidence that is consistent with these loci having been horizontally transferred from the alphaproteobacteria into both the beta and gamma-proteobacteria. ABC transporters within these loci adopt 2 unique genetic arrangements, and although biological data suggests they are functional erythritol transporters, phylogenetic analysis suggests they may not be orthologs and probably should be considered analogs. Finally, evidence for the presence of paralogs, and xenologs of erythritol catabolic genes in some of the genomes included in the analysis is provided.
\end{abstract}

\section{Background}

Operons are multigene arrangements transcribed as a single mRNA and are one of the defining features found in bacterial and archaeal genomes. This arrangement allows genes to be co-regulated, and members of operons are usually involved in the same functional pathway [1,2]. Although operons are prominent features in the genomes of bacteria and archaea, the evolution and mechanisms that promote operon formation are still not resolved and a number mechanisms have been proposed [3-8]. These mechanisms involve dynamic genetic events that include gene transfer events, deletions, duplications, and recombinations $[2,5,8]$. Since operons

\footnotetext{
* Correspondence: oresniki@cc.umanitoba.ca

Department of Microbiology, University of Manitoba, Winnipeg, MB R3T 2N2, Canada
}

\section{Biomed Central}

(c) 2013 Geddes et al.; licensee BioMed Central Ltd. This is an Open Access article distributed under the terms of the Creative Commons Attribution License (http://creativecommons.org/licenses/by/2.0), which permits unrestricted use, distribution, and reproduction in any medium, provided the original work is properly cited. are prominent features in bacterial genomes, and often encode genes with metabolic potential, it may be assumed that their evolution is under some selection pressure, thus allowing prokaryotic cells to rapidly adapt, compete and grow under changing environmental conditions.

The metabolic capability of an organism can be a function of its genome size and gene complement and these greatly affect its ability to live in diverse environments. The alpha subdivision of the proteobacteria includes some organisms that are very similar phylogenetically but inhabit many diverse ecological niches, including a number of bacteria that can interact with eukaryotic hosts [9]. The genome sizes of these organisms varies from about $1 \mathrm{MB}$ for members of the genus Rickettsia to approximately $9 \mathrm{MB}$ for members of the bradyrhizobia 
[10]. Comparative genomic studies of this group has led to the supposition that there has been two independent reductions in genomic size, one which gave rise to the Brucella and Bartonella, the other which gave rise to the Rickettsia [11]. In addition, it also suggests that there has been a major genomic expansion and that roughly correlates with the soil microbes within the order Rhizobiales [11]. The genomes of Rhizobia are dynamic. Phylogenetic analysis of 26 different Sinorhizobium and Bradyrhizobium genomes recently showed that recombination has dominated the evolution of the core genome in these organisms, and that vertically transmitted genes were rare compared with genes with a history of recombination and lateral gene transfer [12]. In this manuscript we have utilized comparative genomics in a focused manner to investigate the evolution of genes and loci involved in the catabolism of the sugar alcohols erythritol, adonitol and L-arabitol, primarily within the alpha-proteobacteria.

The number of bacterial species that are capable of utilizing the common 4 carbon polyol, erythritol, as a carbon source is restricted [13]. Catabolism of erythritol has been shown to be important for competition for nodule occupancy in Rhizobium leguminosarum as well as for virulence in the animal pathogen Brucella suis [14]. Genetic characterization of erythritol catabolic loci has only been performed in $R$. leguminosarum, $B$. abortus and Sinorhizobium meliloti. In these organisms erythritol is broken down to dihydroxyacetonephosphate using the core erythritol catabolic genes eryABC-tpiB [15]. During characterization of the erythritol locus of $S$. meliloti, it was observed that despite the close homologies of core erythritol genes, the genetic content and arrangement of the locus was drastically different from the previously characterized loci of $B$. abortus and R. leguminosarum [16]. In particular the locus encodes the catabolism of two 5-carbon pentitols (adonitol and L-arabitol) in addition to erythritol. It was shown that the $\mathrm{ABC}$ transporter encoded by mptABCDE and erythritol kinase encoded by ery $A$ can also be used for adonitol and L-arabitol, and several genes in the locus are involved in adonitol and L-arabitol, but not erythritol catabolism including lalA-rbtABC [15].

The differences between the erythritol loci in the sequenced S. meliloti strain Rm1021 [17], and R. leguminosarum, led us to question what the relationship of these erythritol catabolic loci may be to other putative erythritol catabolic loci in bacterial species. In this work we focus on this question by analyzing the content and synteny of loci containing homologs to the erythritol genes in other sequenced organisms. The results of the analysis lend support to several hypotheses regarding operon evolution, and in addition, the data predicts loci that may be involved in polyol transport and metabolism in other proteobacteria.

\section{Methods}

\section{Identification of erythritol loci}

The data set of erythritol loci utilized in this work was constructed in a two-step process. First BLASTN was used to identify sequenced genomes containing homologs to the core erythritol catabolic genes $R$. leguminosarum and S. meliloti [18]. The use of BLASTN rather than BLASTP at this stage allowed us to refine the search to bacteria with sequenced genomes. Furthermore, limiting the search to genes with highly similar sequences by using BLASTN allowed us to limit our search to only genes that are likely involved in erythritol catabolism, since all of these genes encode proteins in highly ubiquitous families found throughout bacterial genomes. Initially BLASTN searches were performed using all the core erythritol genes shared between $R$. leguminosarum and $S$. meliloti $(\operatorname{ery} A, \operatorname{ery} B, \operatorname{ery} C$ and $\operatorname{ery} D)$. However, the search using ery $A$ provided the most diverse data set that also showed a sharp drop in E-value and query coverage. Using either eryA from R. leguminosarum, or eryA from S. meliloti for the BLASTN search resulted in an identical data set. Genomes containing homologs to ery $A$ were selected on the basis of E-values less than 1.00E-5. In cases where multiple strains of the same bacterial species were found to have highly homologous putative erythritol genes (>99\% identity) only a single representative of the species was used to avoid redundancy. Additionally $B$. melitensis $16 \mathrm{M}$ and $B$. suis 1330 were chosen as representatives of the Brucella lineage despite a large number of Brucella species that were identified in our search due to the high degrees of similarity between their erythritol catabolic genes.

Second, the genetic region containing ery $A$ in these organisms was identified and analyzed using the IMG Ortholog Neighborhood Viewer (http://img.jgi.doe.gov) [19] in order to construct the gene maps (loci). The amino acid sequence of EryA from S. meliloti was used as a query for the IMG Ortholog Neighborhood Viewer search.

To analyze the genetic content of organisms in our data set, the amino acid sequence encoded by each gene involved in erythritol catabolism in R. leguminosarum, or in erythritol, adonitol or L-arabitol catabolism in $S$. meliloti, was individually used in a BLASTP search of the 19 genomes in the data set. The sugar binding proteins of the S. meliloti and R. leguminosarum transporter were used as representatives of the entire $A B C$ transporter. Identity cut-off values that were used to delineate potential homologs to erythritol proteins were unique to each query amino acid sequence. Cut-off values were as follows: MptA: 56\%, EryD: 44\%, EryA: 46\%, RbtA: 50\%, EryB: 65\%, LalA: 49\%, RbtB: 51\%, RbtC: 40\%, EryC: 68\%, TpiB: 69\%, EryR: 61\%, EryG: 73\%. These values were manually determined and generally 
correlated to a large drop in percentage identity within the BLASTP hits.

Homologs identified that were not within the primary eryA containing loci were used as a query within IMGOrtholog neighborhood viewer to analyze the region surrounding them. Secondary loci containing homologs to some of these genes were identified in Mesorhizobium sp. and Sinorhizobium fredii. These loci are putative erythritol loci based on homology to known loci involved in erythritol catabolism in Sinorhizobium meliloti [15,16], Rhizobium leguminosarum [20] and Brucella abortus[21]. Despite not having been experimentally verified we will refer to all loci in our data set as erythritol loci for the purpose of this manuscript.

\section{Phylogenetic analysis}

Amino acid sequences of homologs to proteins previously shown to play a role in erythritol, adonitol or L-arabitol catabolism from each of the organisms in the data set were collected and used for phylogenetic analysis. The $16 \mathrm{~S}$ $r D N A$ and RpoD sequences were also extracted from the NCBI database for species examined in this study in order to obtain a potential species tree that could be compared with the various phylogenetic gene trees obtained from the individual genes located within the polyol (i.e. erythritol, arabitol, and adonitol) utilization loci. Amino acid sequences were aligned using Clustal-X [22] and PRALINE [23] the resulting alignments were refined manually with the GeneDoc program v2.5.010 [24].

Phylogenies were generated with maximum likelihood analysis (ML) as implemented in the Molecular Evolutionary Genetic Analysis package (MEGA5) [25] and with MrBayes [26]. MEGA5 was used to identify the most suitable substitution models for the aligned data sets. In order to evaluate support for the nodes observed in the ML phylogenetic trees bootstrap analysis [27] was conducted by analysing 1000 pseudo replicates.

The MrBayes program (v3.1) was used for Bayesian analysis [26,28] and the parameters set for amino acid alignments were mixed models and for the 16S rDNA gamma distribution with 4 rate categories. The models used (setting mixed model) for generating the final 50\% majority rule trees were estimated by the program itself. The Bayesian inference of phylogenies was initiated from a random starting tree and four chains were run simultaneously for 1000000 generations; trees were sampled every 100 generations. The first $25 \%$ of trees generated were discarded ("burn-in") and the remaining trees were used to compute the posterior probability values.

Phylogenetic trees were constructed for RpoD, 16S rDNA and all the key genes associated with the EryA genes. Phylogenetic trees were plotted with the TreeView program [29] using MEGA5 and/or MrBayes tree outfiles. Final trees were annotated using Adobe Illustrator.

\section{Results}

Phylogenetic distribution of putative erythritol loci

Based on homology to eryA from Sinorhizobium meliloti and Rhizobium leguminosarum we have compiled a data set of 19 different putative erythritol loci from 19 different proteobacteria (Table 1). Previous studies suggested that erythritol loci may be restricted to the alpha-proteobacteria [20]. While a majority of the erythritol loci we identified followed this scheme, surprisingly we identified putative erythritol catabolic loci in Verminephrobacter eiseniae (a beta-proteobacterium) and Escherichia fergusonii (a gamma-proteobacterium). Erythritol loci are not widely distributed through the alpha-proteobacteria. A majority of the loci we identified were within the order Rhizobiales. Outside of the Rhizobiales we also identified erythritol loci in Acidiphilium species and Roseobacter species. Within the Rhizobiales, erythritol loci were notably absent from a large number of bacterial species such as Rhizobium etli, Agrobacterium tumefaciens and Bradyrhizobium japonicum that are closely related to other species that we have identified that contain erythritol loci. We also note that erythritol loci appear to be plasmid localized only in $S$. fredii and $R$. leguminosarum. In all other cases the loci appear to be found on chromosomes.

\section{Genetic content of loci}

The genetic content of each of the organisms ery loci were analyzed by conducting a BLASTP search to the 19 genomes in our data set of the amino acid sequence of each gene associated with erythritol catabolism in $R$. leguminosarum, or erythritol, adonitol or L-arabitol catabolism in S. meliloti. The results of the BLAST search are presented in Table 2, depicting the presence or absence of homologs to erythritol, adonitol or L-arabitol catabolic genes in each of the genomes that was investigated. Gene maps of erythritol loci were constructed based on the output of our IMG Ortholog Neighborhood Viewer searches and are depicted in Figure 1.

Genes encoding homologs to the core erythritol proteins EryA, EryB and EryD were ubiquitous throughout our data set (Table 2). With respect to the remaining genes, the genetic content of the species can be grouped into three broad categories. (1) Species that contain genes encoding homologs associated with erythritol, adonitol and L-arabitol catabolism. This includes $S$. meliloti, S. medicae, S. fredii, M. loti, M. opportunism, $M$. ciceri, $R$. denitrificans and $R$. litoralis. These genomes contained homologs to genes that encode enzymes specifically involved erythritol catabolism such as EryC, and $\mathrm{TpiB}$ as well as specifically involved in adonitol and $\mathrm{L}$-arabitol catabolism including LalA, and RbtBC. They also contain genes encoding an $\mathrm{ABC}$ transporter homologous to the $S$. meliloti erythritol, adonitol and L-arabitol transporter (MptABCDE) and do not encode 
Table 1 Bacterial genomes used in this study containing erythritol loci

\begin{tabular}{|c|c|c|}
\hline Genome & Accession number & Reference/ Affiliation \\
\hline Sinorhizobium meliloti 1021 & AL591688.1 & {$[17]$} \\
\hline Sinorhizobium medicae WSM419 & СР000738.1 & {$[30]$} \\
\hline Sinorhizobium fredii NGR234 & СР000874.1 & [31] \\
\hline Mesorhizobium opportunism WSM2075 & СР002279.1 & US DOE Joint Genome Institute \\
\hline Mesorhizobium loti MAFF303099 & BA000012.4 & {$[32]$} \\
\hline Mesorhizobium ciceri bv. biserrulae WSM1271 & СР002447.1 & US DOE Joint Genome Institute \\
\hline Bradyrhizobium sp. BTAi1 & СР000494.1 & [33] \\
\hline Bradyrhizobium sp. ORS278 & CU234118.1 & [33] \\
\hline Agrobacterium radiobacter $\mathrm{K} 84$ & СР000629.1 & [34] \\
\hline Ochrobactrum anthropi ATCC 49188 & СР000759.1 & {$[35]$} \\
\hline Brucella suis 1330 & СР002998.1 & {$[36]$} \\
\hline Brucella melitensis $16 \mathrm{M}$ & AE008918.1 & {$[37]$} \\
\hline Acidiphilium multivorum AlU301 & AP012035.1 & NITE Bioresource Information Center \\
\hline Acidiphilium cryptum JF-5 & СР000697.1 & US DOE Joint Genome Institute \\
\hline Roseobacter denitrificans Och114 & CP000362.1 & {$[38]$} \\
\hline Roseobacter litoralis Och149 & CP002623.1 & [39] \\
\hline Rhizobium leguminosarum bv. viciae 3814 & AM236086.1 & [40] \\
\hline Rhizobium leguminosarum bv. trifolii WSM1325 & СР001623.1 & [41] \\
\hline Verminephrobacter eiseniae EF01-2 & СР000542.1 & US DOE Joint Genome Institute \\
\hline Escherichia fergusonii ATCC 35469 & CU928158.2 & Genoscope - Centre National de Sequencage \\
\hline
\end{tabular}

homologs to the $R$. leguminosarum erythritol transporter (EryEFG). One notable exception is $M$. ciceri which encodes EryEFG homologs rather than MptABCDE (Table 2). (2) Species that contain all the genes associated with erythritol catabolism, but lack the genes associated with adonitol or L-arabitol catabolism. These species include $R$. leguminosarum bvs. viciae and trifolii, A. radiobacter, O. anthropi, B. suis, B. melitensis, and E. fergusonii. These loci encode EryABCDR-TpiB as well as homologs to the $R$. leguminosarum ABC transporter EryEFG, but lack genes encoding homologs to enzymes associated specifically with adonitol and L-arabitol catabolism or the S. meliloti transport protein MptABCDE. E. fergusonii contains the most minimal set of homologs to erythritol genes of all the genomes investigated, and did not encode EryR and TpiB. (3) Species that do not encode the specifically erythritol associated EryC, EryR, and TpiB, but encode the adonitol/L-arabitol catabolic complement LalA-RbtABC and homologs to the $S$. meliloti polyol transporter MptABCDE. These include Bradyrhizobium spp. BTAi1 and ORS278, A. multivorum, A. cryptum and V. eiseniae.

\section{The genetic structure of erythritol loci}

The genetic context of eryA in each of the genomes in our data set supported that each of these organisms contained an erythritol locus. A physical map of the loci in each of these organisms is depicted in Figure 1. Of note, a number of putative erythritol loci were identified in organisms with incomplete genome sequences at the time of analysis, and thus are not discussed here, including: Octadecabacter antarcticus, Pelagibaca bermudensis Enterobacter hormaechei, Fulvimarina pelagi, Aurantimonas sp. SI85-9A1, Roseibium sp. TrichSKD4, Burkholderia thailandensis and Stappia aggregata.

The putative erythritol loci of bacteria in our data set ranged in genetic complexity with the loci from $S$. meliloti and S. medicae containing 17 different genes, to the simplest being the locus of E. fergusonii, which contained only two divergently transcribed operons that are homologous to the eryEFG and eryABCD loci of $R$. leguminosarum. A number of species contained loci that were identical in content and arrangement to the $R$. leguminosarum erythritol locus including members of the Brucella, Ochrobacterum, and Agrobacterium. The only species that contains a locus identical in content and arrangement to $S$. meliloti is the closely related Sinorhizobium medicae. The locus of Sinorhizobium fredii NGR234, contains all but one of the genes (fucA1) found in the other Sinorhizobium loci (Figure 2).

The loci of Mesorhizobium species were varied, however all three Mesorhizobium sp. contained an independent locus with homologs to lalA and $r b t B C$ elsewhere in the genome (Figure 1). Interestingly, while Mesorhizobium loti 
Table 2 Content of putative erythritol loci

\begin{tabular}{|c|c|c|c|c|c|c|c|c|c|c|c|c|}
\hline \multirow[t]{2}{*}{ Genome } & \multicolumn{12}{|c|}{ Homologs involved in erythritol, adonitol and/or L-arabitol catabolism } \\
\hline & EryA & EryB & EryD & EryC & EryG & EryR & TpiB & MptA & LalA & RbtA & RbtB & RbtC \\
\hline Sinorhizobium meliloti & + & + & + & + & - & + & + & + & + & + & + & + \\
\hline Sinorhizobium medicae & + & + & + & + & - & + & + & + & + & + & + & + \\
\hline Sinorhizobium fredii & + & + & + & + & - & ++ & ++ & + & + & + & + & + \\
\hline Mesorhizobium opportunism & + & + & + & + & - & + & + & + & + & + & + & + \\
\hline Mesorhizobium loti & + & + & + & + & - & + & + & + & ++ & + & + & + \\
\hline Mesorhizobium ciceri bv. biserrulae & + & + & + & + & + & - & + & - & + & - & + & + \\
\hline Roseobacter denitrificans & + & + & + & + & - & - & + & + & + & + & + & + \\
\hline Roseobacter litoralis & + & + & + & + & - & - & + & + & + & + & + & + \\
\hline Rhizobium leguminosarum bv. viciae & + & + & + & + & + & + & + & - & - & - & - & - \\
\hline Rhizobium leguminosarum bv. trifolii & + & + & + & + & + & + & + & - & - & - & - & - \\
\hline Agrobacterium radiobacter & + & + & + & + & + & + & + & - & - & - & - & - \\
\hline Ochrobacterum anthropi & + & + & + & + & + & + & + & - & - & - & - & - \\
\hline Brucella suis 1330 & + & + & + & + & + & + & + & - & - & - & - & - \\
\hline Brucella melitensis 16M & + & + & + & + & + & + & + & - & - & - & - & - \\
\hline Escherichia fergusonii & + & + & + & + & + & - & - & - & - & - & - & - \\
\hline Bradyrhizobium sp. BTAi1 & + & + & + & - & - & - & - & + & + & + & + & + \\
\hline Bradyrhizobium sp. ORS278 & + & + & + & - & - & - & - & + & + & + & + & + \\
\hline Acidiphilium multivorum & + & + & + & - & - & - & - & + & + & + & + & + \\
\hline Acidiphilium cryptum & + & + & + & - & - & - & - & + & + & + & + & + \\
\hline Verminephrobacter eiseniae & + & + & + & - & - & - & - & + & + & + & + & + \\
\hline
\end{tabular}

+ indicates presence of homolog in the genome, - indicates absence of homolog in the genome, ++ indicates presence of 2 homologs in genome.

and Mesorhizobium opportunism both contain transporters homologous to mptABCDE, Mesorhizobium ciceri bv. biserrulae contains a transporter homologous to eryEFG. This operon also contains the same hypothetical gene that is found at the beginning of the $R$. leguminosarum eryEFG transcript. The transporters however, are arranged in a manner similar to that seen in $S$. meliloti and the gene encoding the regulator ery $D$, is found ahead of the transporter genes, whereas in $R$. leguminosarum and Brucella, eryD is found following eryC (Figure 1). We also note that whereas $M$. loti and $M$. opportunism both contain a putative fructose 1,6 bis phosphate aldolase gene between the eryR-tpiB-rpiB operon and eryC, a homolog to this is also gene is found adjacent to the rpiB in Brucella.

Bradyrhizobium sp. BTAi1, and ORS278, A. cryptum and $V$. eiseniae all have similar genetic arrangement to that of $S$. meliloti, except that they do not contain a homolog to eryC, or an associated eryR-tpiB-rpiB operon. These loci also differ primarily in their arrangement of lalA-rbtBC (Figure 1).

\section{The phylogenies of erythritol proteins do not correlate with species phylogeny}

The DNA sequences of $16 \mathrm{~S} r D N A$ (data not shown) as well as the amino acid sequences of RpoD were extracted from
GenBank to analyze the phylogenetic relationships of the organisms examined in this study, using the most phylogenetically distant organism Verminephrobacter eiseniae as an out-group. The results of the $16 \mathrm{~S} r D N A$ and RpoD sequence analyses were in concordance with each other and are consistent with phylogenies that have been previously generated [42]. Initial comparison of the operon structures with the generated phylogenies suggested that the operon structure(s) did not correlate with the species phylogeny. Since the structure of some operons did not correspond well with the species phylogenies we wished to determine if operon structure did correlate with any of the erythritol genes found at the $S$. meliloti loci. Since homologs to EryA, EryB and EryD were ubiquitous through the data set, it was decided to construct phylogenies based on Maximum Likelihood and Bayesian analysis using the EryA, EryB and EryD data sets. The topology of the phylogenetic tree using EryA is presented in Figure 2. A tree including branch lengths is included as Additional file 1: Figure S1. $V$. eiseniae was also the most distant member with respect to the EryA phylogeny and again used as an outgroup. The phylogenetic trees of EryB and EryD are not shown but were generally consistent with the EryA phylogeny. The species tree, based on RpoD, was included as a mirror tree with the EryA tree to demonstrate possible horizontal gene transfer events (Figure 2). 


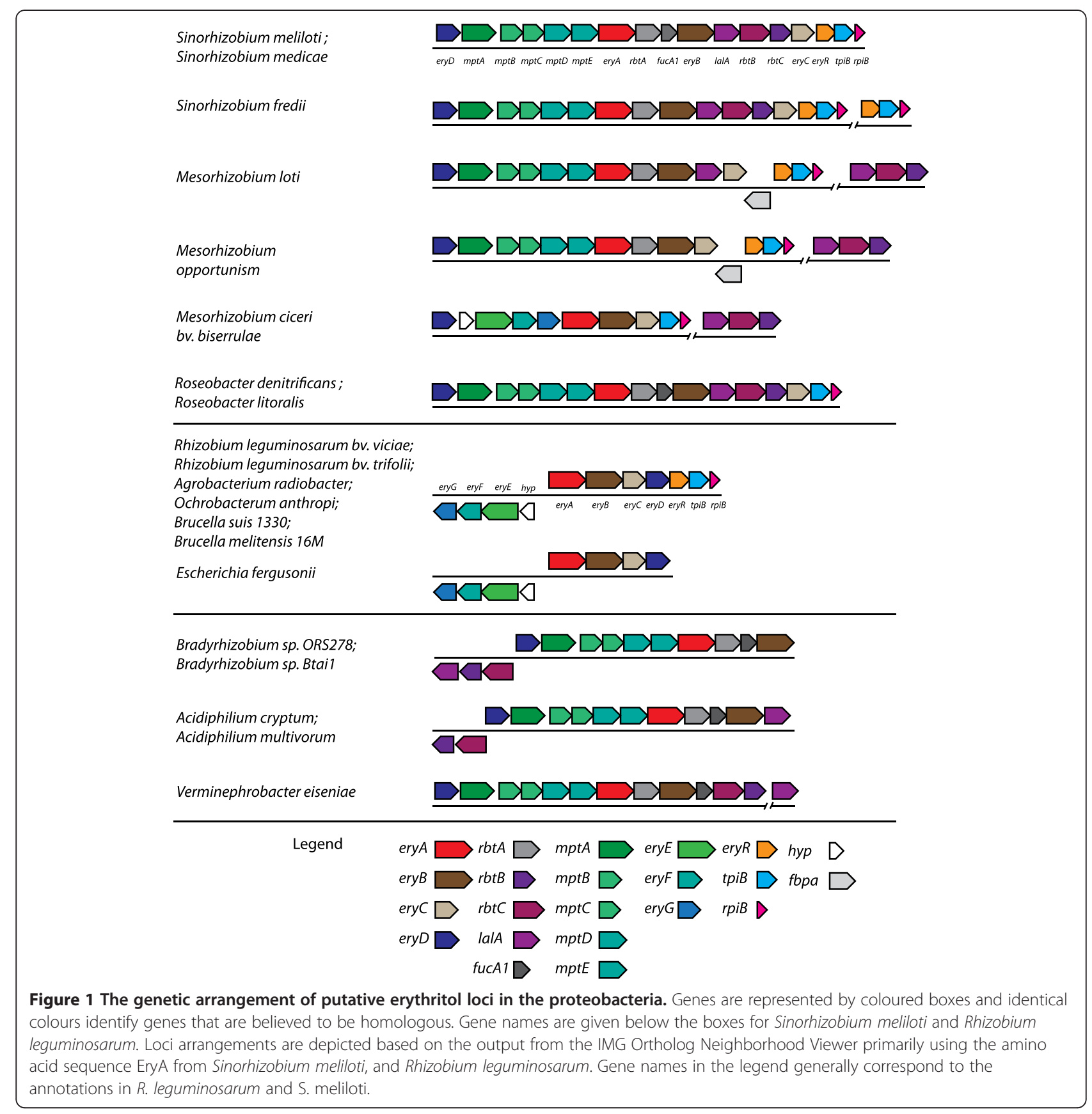

The data show that there is a high degree of correlation between the loci configuration and the EryA phylogenetic tree (Figure 1,2). We note the similarity of the loci of $A$. radiobacter and $R$. leguminosarum to Brucella species and $O$. anthropi but not to the more closely related Sinorhizobium species. This suggests that a horizontal gene transfer may have occurred between these organisms. This is in agreement with what has been previously reported [20]. It also seems likely that a horizontal gene transfer event may have occurred between the Brucella and E. fergusonii. This may explain the unique occurrence of the loci's presence in a member of the gamma-proteobacteria. Finally, our mirror tree suggests that a horizontal gene transfer of the more complex erythritol locus may have occurred between $M$. loti and an ancestral species the Sinorhizobium species (Figure 2).

\section{Modes of evolution for the polyol utilization loci}

Comparison of the phylogenetic trees of EryA, EryB and EryD to the arrangement and content of the loci led us to more thoroughly investigate the phylogenies of a 


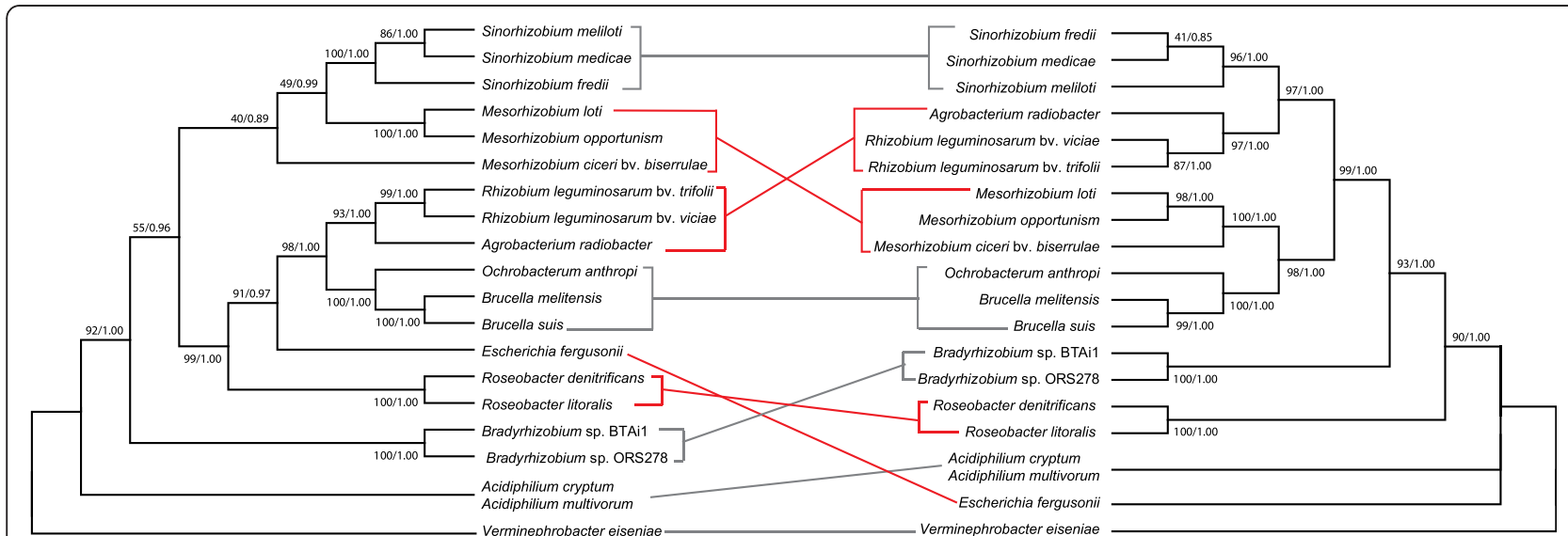

Figure 2 The phylogenetic tree of erythritol proteins does not correlate with species phylogeny; evidence for horizontal gene transfer. EryA phylogenetic tree (Left) and RpoD species tree (Right) were constructed using ML and Bayesian analysis. Support for each clade is expressed as a percentage (Bayesian/ML, ie. posterior probability and bootstrap values respectively) adjacent to the nodes that supports the monophyly of various clades. V. eiseniae was used as an outgroup for both trees since it was the most phylogenetically distant organism. A tree including branch lengths for EryA is included as Additional file 1: Figure S1.

number of proteins that stood out as unique within the data set. These phylogenies have led us to postulate modes of evolution that may have occurred in these loci.

BLASTP analysis showed a clear distinction between the type of transporter encoded by each of the loci and the remaining genetic content. In general, loci that contained adonitol/L-arabitol type genes contained a transporter homologous to the S. meliloti MptABCDE (Table 2, Figure 1). Loci that contained only erythritol genes contained a transporter homologous to the EryEFG of $R$. leguminosarum. One exception to this correlation was $M$. ciceri bv. biserrulae which contained a homologous transporter to EryEFG rather than MptABCDE. This is interesting because $M$. ciceri groups with the other Mesorhizobia in the EryABD trees. In order to analyze the evolution of these transporters more clearly, phylogenetic trees were constructed of homologs to EryG and homologs to MptA (Figure 3). In general the phylogenies are in agreement with the EryABD phylogenies, with the exception of $M$. ciceri which falls on a basal branch of the EryG phylogeny. The disparities between the EryG and EryABD phylogenies of $M$ ciceri strongly suggest that parts of its erythritol locus have a different origin. This may have been the result of horizontal gene transfer of a second $R$. leguminosarum type erythritol locus, followed by recombination between the two.

In two organisms, apparent duplications of genes were present. In M. loti one homolog of lalA was present in the erythritol locus, while a second copy was present elsewhere in the genome adjacent to homologues of $r b t B$ and $r b t C$, consistent with its location in the other two Mesorhizobium genomes. In S. fredii homologs to the apparent small operon that contains eryR-tpiB-rpiB were found both, as expected, in the erythritol locus, but also elsewhere on the chromosome in the same arrangement. To analyze the evolutionary history of these duplications phylogenetic trees were constructed for the LalA and TpiB homologs (Figure 4 and 5). The two copies of the lalA gene in $M$. loti are most likely an example of paralogs, as they still group within the same clade among other lalA homologs (Figure 4). The tpiB genes (Figure 5) in S. fredii are possible examples of xenologs [43] as the phylogenetic tree shows that the two versions of the tpiB gene in S. fredii are only distantly related, with one homolog grouping within the expected clade that includes S. medicae and S. meliloti and the second homolog (not part of the main locus) showing monophyly with those found in a clade containing $R$. leguminosarum sp., B. suis, etc. (Figure 5).

\section{Discussion}

A number of models that are not mutually exclusive have been proposed to account for the formation and evolution of operons. Two broad aspects need to be considered, transfer of genes between organisms, as well as gathering and distributing genes within a genome. There is strong support for horizontal gene transfer as a driving force for evolution of gene clusters [44]. More recently, it has been shown that genes acquired by horizontal gene transfer events appear to evolve more quickly than genes that have arisen by gene duplication events [45]. Within a genome the "piece-wise" model suggests that complex operons can evolve through the independent clustering of smaller "suboperons" due to selection pressures for the optimization for equimolarity and co-regulation of gene products [6]. Finally it has been suggested that the final stages of operon building can be the loss of "ORFan" genes $[4,6]$. 

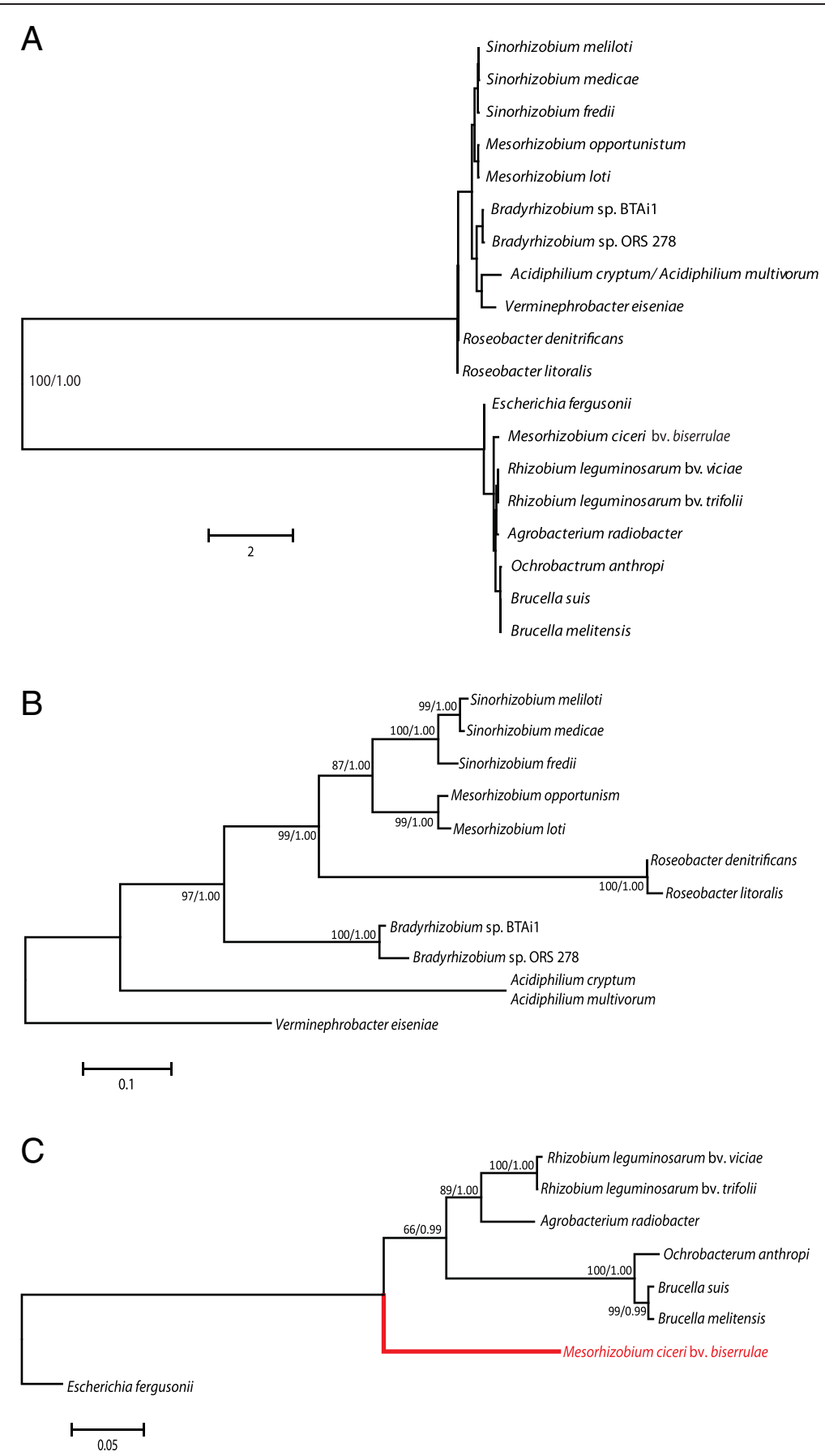

Figure 3 Phylogenetic trees of erythritol transporters. Unrooted phylogenetic tree including putative homologues to the sugar binding protein MptA of Sinorhizobium meliloti and EryG of Rhizobium leguminosarum (A). Support is provided for the node that clearly separates the putative homologues into two distinct and distant clades. Separate phylogenetic trees for erythritol transporters homologous to MptABCDE and EryEFG are depicted (B and $\mathbf{C}$ ) using aligned amino acid sequences of the putative sugar binding proteins MptA (B) and EryG (C) as representatives of the transporters phylogenies. The branch that shows the anomalous placement of the Mesorhizobium ciceri bv. biserrulae within the tree of EryEFG homologs is highlighted in red. Trees were constructed using ML and Bayesian analysis. Support for each node is expressed as a percentage based on posterior probabilities (Bayesian analysis) and bootstrap values (ML). The branch lengths are based on $\mathrm{ML}$ analysis and are proportional to the number of substitutions per site. 


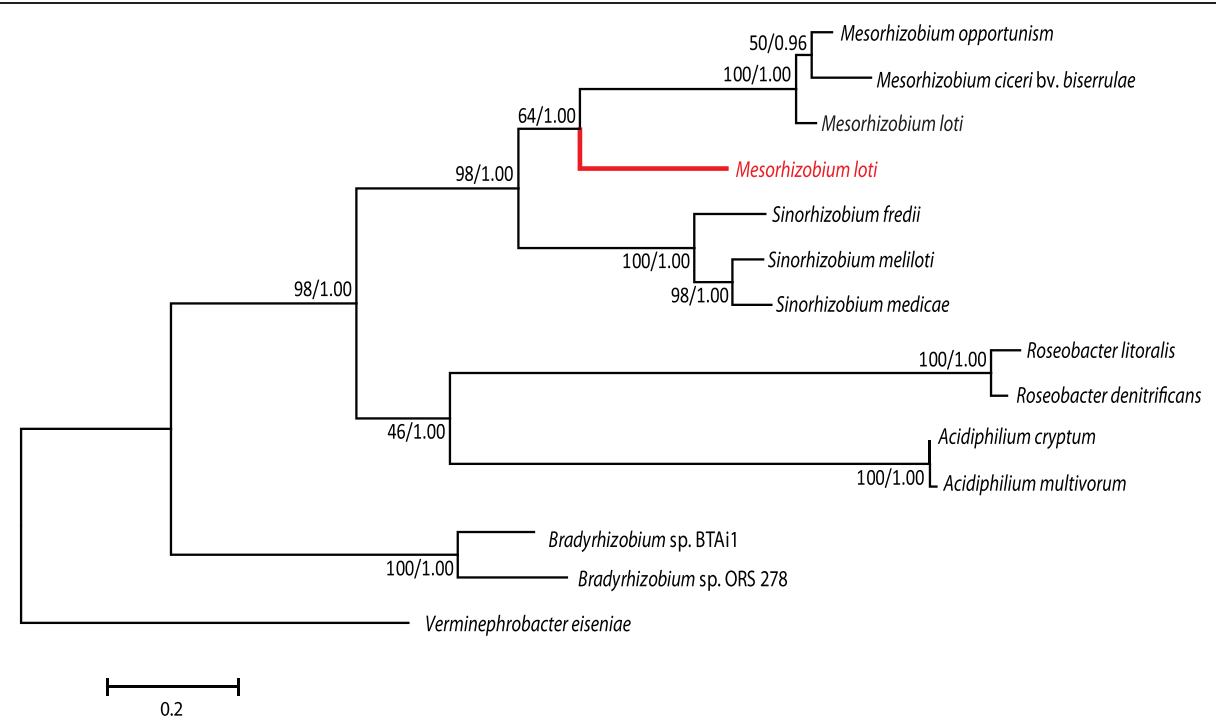

Figure 4 Mesorhizobium loti contains paralogs of LalA. The phylogeny of the L-arabitol catabolic gene LalA is depicted. Mesorhizobium loti contains a copy of lalA within an independent suboperon like the other Mesorhizobium species, as well as a second lalA homolog within the erythritol locus (Figure 1). The branch corresponding to the additional homolog within the erythritol locus is highlighted in red. The tree was constructed using ML and Bayesian analysis. Support for each node is expressed as a percentage based on posterior probabilities (Bayesian analysis) and bootstrap values (ML). The branch lengths are based on ML analysis and are proportional to the number of substitutions per site.

The data presented here provide examples supporting these models of operon evolution. The components of the polyol catabolic loci we have identified have been involved in at least 3 horizontal gene transfers within the proteobacteria (Figure 2). In addition, components such as the transporter eryEFG have been moved from the $R$. leguminosarum clade of loci into the $M$. ciceri bv. biserrulae polyol locus (see Figure 3A and 3B). The later species based on its phylogenetic position and category of polyol locus (S. meliloti) would have been expected to contain the $m t p A$ gene. The presence of possible paralogs of lalA (Figure 4) and the presence of tpiB xenologs (Figure 5) are also evidence for duplication and horizontal transfer events. Since $S$. fredii also contains a homolog to tpiA of S. meliloti (data not shown), to our knowledge, this is the only example of an organism

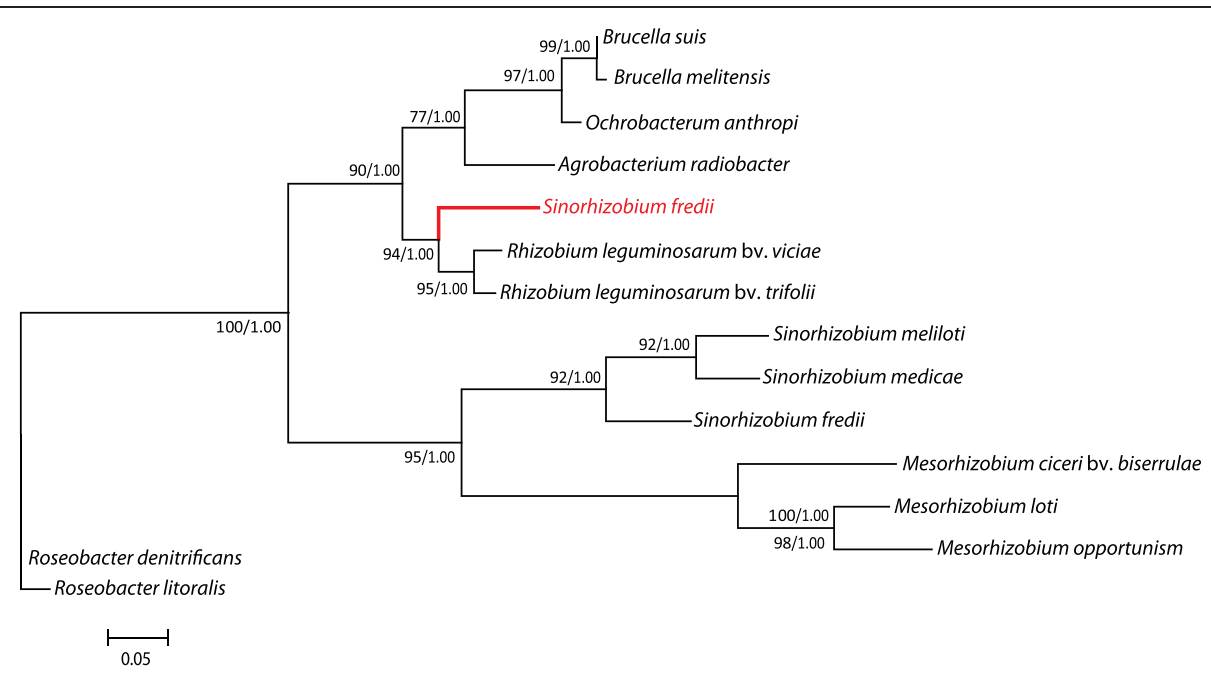

Figure 5 Sinorhizobium fredii encodes TpiB xenologs. Sinorhizobium fredii contains a second suboperon that appears homologous to the eryRtpiB-rpiB suboperon in the erythritol locus (Figure 1). The TpiB amino acid sequence was used as a representative of this suboperon to construct a phylogenetic tree. The branch corresponding to the TpiB encoded outside of the erythritol locus is highlighted in red. The tree was constructed using ML and Bayesian analysis. Support for each node is expressed as a percentage based on posterior probabilities (Bayesian analysis) and bootstrap values (ML). The branch lengths are based on $\mathrm{ML}$ analysis and are proportional to the number of substitutions per site. 
containing three triose-phosphate isomerases (Figure 2, Figure 5).

A striking example of a horizontal gene transfer and genetic rearrangement is exemplified by $M$. ciceri (Figure 1, Figure 2). It is likely that an exchange between M. loti and a common ancestor of S. meliloti, S. medicae and $S$. fredii NGR234 occurred. $M$. loti is located in the same clade as the Brucella and O. anthropi in the species tree (Figure 2). Despite this, M. loti contains many of the genes corresponding to the adonitol and Larabitol type loci of other species that cluster close to the base of the species tree such as Bradyrhizobium spp. (Figure 2). The presence of these factors in addition to the chimeric composition of the M. loti locus leads us to hypothesise that an ancestor of $M$. loti may have contained both an erythritol locus like that of the Brucella as well as a polyol type locus like that seen in the Bradyrhizobia, A. cryptum and V. eiseniae.

The lalA, $r b t B, r b t C$ suboperon appears to be the key component of the polyol locus in the Bradyrhizobium type loci (Figure 1). Among the 19 loci identified, these three genes can be linked into a suboperon, embedded within the main locus (eg. R. litoralis) or split among two transcriptional units (see A. cryptum or $V$. eiseniae). As well, the gene module (or suboperon) eryR, tpiB- rpiB is presumably found in all erythritol utilizing bacteria. The acquisition of this module along with the lalA, $r b t B$ and $r b t C$ suboperon may have allowed for the evolution of the more complex S. meliloti type locus (see Figure 2).

The absence of fucA in S. fredii NGR234 and M. loti appears to be an example of the loss of an "ORFan" gene event having occurred. The gene is still present in $S$. meliloti however it has been shown that it is not necessary for the catabolism of erythritol, adonitol, or L-arabitol [15]. It is likely that it was lost during the divergence of $M$. loti and S. fredii NGR234 from their common ancestors to S. meliloti. If this is true, it may be reasonable to assume that fucA may eventually also be lost from the S. meliloti erythritol locus.

In S. meliloti, erythritol uptake has been shown to be carried out by the proteins encoded by mptABCDE $[15,16]$, whereas in $R$. leguminosarum growth using erythritol is dependent upon the eryEFG [20]. Although both transporters appear to carry out the same function, the phylogenetic analysis clearly shows that they have distinct ancestors and may be best classified as analogues rather than orthologues (Figure 3). In addition, it has been shown that MptABCDE is also capable of transporting adonitol and L-arabitol [15]. We note that these polyols appear to have stereo-chemical identity over three carbons and that EryA of S. meliloti can also use adonitol and L-arabitol as substrates [15]. It is unknown whether EryA from $R$. leguminosarum has the ability to interact with these substrates.
The three distinct groups of loci we have identified probably correspond to the metabolic potential of these regions to utilize polyols. The locus of S. meliloti has been shown to contain the full complement of genes required to confer growth on using both erythritol and adonitol and L-arabitol as sole carbon sources $[15,16]$. Given that S. fredii NGR234 and M. loti each contain homologs to all of these genes, except for fucA which is not necessary for the catabolism of any of the sugars [15], it follows that these two loci may also be capable of catabolising all three polyols. It has also been established that the $B$. abortus and $R$. leguminosarum type loci are used for erythritol catabolism, and given the annotation and degree of relatedness $(E$ value $=0$ ) of proteins belonging to all species in the clade, it is not expected that these loci would be capable of breaking down additional polyols $[20,21]$. This is supported by the fact that the introduction of the $R$. leguminosarum cosmid containing the erythritol locus into $S$. meliloti strains unable to utilize erythritol, adonitol, and L-arabitol were unable to be complemented for growth on adonitol and L-arabitol [15]. It is however necessary to remember that some of identified loci are only correlated with polyol utilization based on our analysis and that basic biological function, such as the ability to utilize these polyols has not been previously described.

With the advent of newer generations of sequencing technologies a greater number of bacterial genomes will be sequenced. It is likely that more examples of rearrangements of catabolic loci through bacterial lineages will be observed. Since the ability to catabolize erythritol is found in relatively few bacterial species, operons that encode erythritol and other associated polyols may be ideal models to observe operon evolution.

\section{Conclusions}

In this work we show that there are at least three distinct erythritol/polyol loci arrangements. Two distinct $\mathrm{ABC}$ transporters can be found within these within these loci and phylogenetic analysis suggests these should be considered analogs. Finally we provide evidence that suggest that these loci have been horizontally transferred from the alpha-proteobacteria into both the beta and gamma-proteobacteria.

\section{Additional file}

Additional file 1: Figure S1. EryA phylogenetic tree was constructed using ML and Bayesian analysis. Support for each clade is expressed as a percentage (Bayesian / ML, ie. posterior probability and bootstrap values respectively) adjacent to the nodes that supports the monophyly of various clades. The branch lengths are based on $\mathrm{ML}$ analysis and are proportional to the number of substitutions per site. This phylogenetic tree was used in the mirror tree in Figure 2 without branch lengths due to space restrictions. 


\section{Competing interests}

The authors declare that they have no competing interests.

\section{Authors' contribution}

BAG collected the data set, performed the analysis and contributed to writing of the manuscript. GH provided advice and assistance with the analysis as well as contributed to the writing of the manuscript. IJO provided advice for the analysis and contributed to the writing of the manuscript. All authors read and approved the final manuscript.

\section{Acknowledgments}

This work was funded by NSERC Discovery Grants to IJO and GH. BAG was funded by an NSERC CGS-D. The authors would like to thank the anonymous reviewer's suggestions that greatly improved the manuscript.

Received: 10 August 2012 Accepted: 20 February 2013

Published: 23 February 2013

\section{References}

1. Omata T, Price GD, Badger MR, Okamura M, Gohta S, Ogawa T: Identification of an ATP-binding cassette transporter involved in bicarbonate uptake in the cyanobacterium Synechoccus sp. strain PCC 7942. Proc Natl Acad Sci USA 1999, 96:13571-13576.

2. Osbourn AE, Field B: Operons. Cell Mol Life Sci 2009, 66:3755-3775.

3. Omelchenko MV, Makarova KS, Wolf Yl, Rogozin IB, Koonin EV: Evolution of mosaic operons by horizontal gene transfer and gene displacement in situ. Genome Biol 2003, 4:R55.

4. Fani R, Brilli M, Lio P: The origin and evolution of operons: the piecewise building of the proteobacterial histidine operon. J Mol Evol 2005, 60:370-390.

5. Price MN, Arkin AP, Alm EJ: The life-cycle of operons. PLoS Genet 2006, 2:e96.

6. Fondi M, Emiliani G, Fani R: Origin and evolution of operons and metabolic pathways. Res Microbiol 2009, 69:512-526.

7. Homma K, Fukuchi S, Gojobori T, Nishikawa K: Gene cluster analysis method identifies horizontally transferred genes with high reliability and indicates that they provide the moain mechanis of operon gain in 8 species of gamma proteobacteria. Mol Biol Evol 2007, 24:805-813.

8. Muzzi A, Moschioni M, Covacci A, Rappuoli R, Donati C: Streptococcus pneumoniae is driven by positive selection and recombination. PLoS One 2008, 3:e3660

9. Kuykendall LD, Shao JY, Hartung JS: Conservation of gene order and content in the circular chromosomes of Candidatus Liberbacter asiaticus and other Rhizobiales. PLoS One 2012, 74:e34673.

10. Batut J, Andersson SGE, O'Callaghan D: The evolution of chronic infections strategies in the alpha-proteobacteria. Nat Rev Microbiol 2004, 2:933-945.

11. Boussau B, Karlberg EO, Frank AC, Legault B, Andersson SGE: Computational inference of scenarios for alpha-proteobacterial genome evolution. Proc Natl Acad Sci USA 2004, 101:9722-9727.

12. Tian CF, Zhou YJ, Zhang YM, Li QQ, Zhang YZ, Li DF, Wang S, Wang J, Gilbert LB, Li YR, et al: Comparative genomics of rhizobia nodulating soybean suggests extensive recruitment of lineage-specific genes in adaptations. Proc Natl Acad Sci USA 2012, 109:8629-8634.

13. Wawskiewicz EJ, Barker HA: Erythritol metabolism by Propionibacterium pentosaceum. J Biol Chem 1968, 243:1948-1956.

14. Burkhardt S, Jiménez de Bagüés MP, Liautard JP, Kohler S: Analysis of the behaviour of eryC mutants of Brucella suis attenuated in macrophages. Infect Immun 2005, 73:6782-6790.

15. Geddes BA, Oresnik IJ: Genetic characterization of a complex locus necessary for the transport and catabolism of erythritol, adonitol, and Larabitol in Sinorhizobium meliloti. Microbiology 2012, 158(8):2180-2191.

16. Geddes BA, Pickering BS, Poysti NJ, Yudistira H, Collins H, Oresnik IJ: A locus necessary for the transport and catabolism of erythritol in Sinorhizobium meliloti. Microbiol 2010, 156:2970-2981.

17. Galibert F, Finan TM, Long SR, Pühler A, Abola P, Ampe F, Barloy-Hubler F, Barnett MJ, Becker A, Boistard P, et al: The composite genome of the legume symbiont Sinorhizobium meliloti. Science 2001, 293:668-672.

18. Altschul SF, Madden TL, Schäffer AA, Zhang JH, Zhang Z, Miller W, Lipman DJ: Gapped BLAST and PSI-BLAST: a new generation of protein database search programs. Nucleic Acids Res 1997, 25:3389-3402.

19. Markowitz VM, Chen IA, Palaniappan K, Chu K, Szeto E, Grechkin Y, Ratner A, Anderson I, Lykidis A, Mavromatis K, et al: The integrated microbial genomes system: an expanding comparative analysis resource. Nucleic Acids Res 2010, 38(suppl 1):D382-D290.

20. Yost CK, Rath AM, Noel TC, Hynes MF: Characterization of genes involved in erythritol catabolism in Rhizobium leguminosarum bv. viciae. Microbiol 2006, 152:2061-2074.

21. Sangari FJ, Agüero J, García-Lobo JM: The genes for erythritol catabolism are organized as an inducible operon in Brucella abortus. Microbiol 2000, 146:487-495.

22. Thompson JD, Gibson TJ, Plewniak F, Jeanmougin F, Higgins DG: The CLUSTAL$X$ windows interface: flexible strategies for multiple sequence alignment aided by quality analysis tools. Nucleic Acids Res 1997, 25:4876-4882.

23. Simossis VA, Kleinjung J, Heringa J: Homology-extended sequence alignment. Nucleic Acids Res 2005, 33:816-824.

24. Nicholas KB, Nicholas HB Jr, Deerfield DWII: GeneDoc: analysis and visualization of genetic variation. EMBNEW News 1997, 4:14.

25. Tamura K, Peterson D, Peterson ND, Stetcher G, Nei M, Kumar S: MEGA5: molecular evolutionary genetics analysis using maximum likelihood, evolutionary distance, and maximum parsimony methods. Mol Biol Evol 2011, 28:2731-2739.

26. Ronquist F, Huelsenbeck JP: MrBayes 3: bayesian phylogenetic inference under mixed models. Bioinformatics 2003, 19:1572-1574.

27. Felsenstein J: Confidence limits on phylogenies: an approach using the bootstrap. Evolution 1985, 39:783-789.

28. Ronquist F: Bayesian inference of character evolution. Trends Ecol Evol 2004, 19:475-481.

29. Page RDM: TREEVIEW: an application to display phylogenetic trees on personal computers. Comput Appl Biosci 1996, 12:357-358.

30. Reeve W, Chain P, O'Hara G, Ardley J, Nandesena K, Bräu L, Tiwari R, Malfatti $\mathrm{S}$, Kiss H, Lapidus A, et al: Complete genome sequence of the Medicago microsymbiont Ensifer (Sinorhizobium) medicae strain WSM419. Stand Genomic Sci 2010, 2(1):77-86.

31. Schmeisser $C$, Liesegang $H$, Krysciak D, Bakkou N, Le Quéré A, Wollherr A, Heinemeyer I, Morgenstern B, Pommerening-Röser A, Flores M, et al: Rhizobium sp. strain NGR234 possesses a remarkable number of secretion systems. Appl Environ Microbiol 2009, 75(12):4035-4045.

32. Kaneko T, Nakamura Y, Sato S, Asamizu E, Kato T, Sasamoto S, Watanabe A, Idesawa K, Ishikawa A, Kawashima K, et al: Complete genome structure of the nitrogen-fixing symbiotic bacterium Mesorhizobium loti. DNA Res 2000, 7:331-338.

33. Giraud E, Moulin L, Vallenet D, Barbe V, Cytryn E, Avarre JC, Jaubert M, Simon $D$, Cartieaux F, Prin Y, et al: Legumes symbioses: absence of Nod genes in photosynthetic bradyrhizobia. Science 2007, 316(5829):1307-1312.

34. Slater SC, Goldman BS, Goodner B, Setubal JC, Farrand SK, Nester EW, Burr TJ, Banta L, Dickerman AW, Paulsen I, et al: Genome sequences of three agrobacterium biovars help elucidate the evolution of multichromosome genomes in bacteria. J Bacteriol 2009, 191(8):2501-2511.

35. Chain PS, Lang DM, Comerci DJ, Malfatti SA, Vergez LM, Shin M, Ugalde RA, Garcia E, Tolmasky ME: Genome of Ochrobactrum anthropi ATCC 49188 T, a versatile opportunistic pathogen and symbiont of several eukaryotic hosts. J Bacteriol 2011, 193(16):4274-4275.

36. Tae H, Shallom S, Settlage R, Preston D, Adams LG, Garner HR: Revised genome sequence of brucella suis 1330. J Bacteriol 2011, 193(22):6410.

37. DelVecchio VG, Kapatral V, Redkar RJ, Patra G, Mujer C, Los T, Ivanova N, Anderson I, Bhattacharyya A, Lykidis A, et al: The genome sequence of the facultative intracellular pathogen Brucella melitensis. Proc Natl Acad Sci USA 2002, 99(1):443-448.

38. Swingley WD, Sadekar S, Mastrian SD, Matthies HJ, Hao J, Ramos H, Acharya CR, Conrad AL, Taylor HL, Dejesa LC, et al: The complete genome sequence of Roseobacter denitrificans reveals a mixotrophic rather than photosynthetic metabolism. J Bacteriol 2007, 189(3):683-690.

39. Kalhoefer D, Thole S, Voget S, Lehmann R, Liesegang H, Wollher A, Daniel R, Simon M, Brinkhoff T: Comparative genome analysis and genome-guided physiological analysis of Roseobacter litoralis. BMC Genomics 2011, 12(1):324.

40. Young JPW, Crossman LC, Johnston AW, Thomson NR, Ghazoui ZF, Hull KH, Wexler M, Curson ARJ, Todd JD, Poole PS, et al: The genome of Rhizobium leguminosarum has recognizable core and accessory components. Genome Biol 2006, 7:R34.

41. Reeve W, O'Hara G, Chain P, Ardley J, Brau L, Nandesena K, Tiwari R, Copeland A, Nolan M, Han C, et al: Complete genome sequence of Rhizobium leguminosarum bv. trifolii strain WSM1325, an effective microsymbiont of annual Mediterranean clovers. Stand Genomic Sci 2010, 2(3):347-356. 
42. Crossman LC, Castillo-Ramírez S, McAnnula C, Lozano L, Vernikos GS, Acosta JL, Ghazoui ZF, Hernández-Lucas I, Meakin G, Walker AW, et al: A common genomic framework for a diverse assembly of plasmids in the symbiotic nitrogen fixing bacteria. PLoS One 2008, 3:e2567.

43. Koonin EV: Orthologs, paralogs, and evolutionary genomics. Annu Rev Genet 2005, 39:309-338.

44. Lawrence JG, Roth JR: Selfish operons: horizontal transfer may drive the evolution of gene clusters. Genetics 1996, 143:1843-1860.

45. Treangen TJ, Rocha EPC: Horizontal transfer, not duplication, drives the expansion of protein families in prokaryotes. PLoS Genet 2011, 7:e1001284.

doi:10.1186/1471-2180-13-46

Cite this article as: Geddes et al.: Phylogenetic analysis of erythritol catabolic loci within the Rhizobiales and Proteobacteria. BMC Microbiology 2013 13:46.

\section{Submit your next manuscript to BioMed Central and take full advantage of:}

- Convenient online submission

- Thorough peer review

- No space constraints or color figure charges

- Immediate publication on acceptance

- Inclusion in PubMed, CAS, Scopus and Google Scholar

- Research which is freely available for redistribution 